\title{
miR-144 promotes the proliferation and differentiation of bone mesenchymal stem cells by downregulating the expression of SFRP1
}

\author{
LING TANG ${ }^{1}$, WENJUN LU ${ }^{1}$, JIAN HUANG $^{2}, \mathrm{XU} \mathrm{TANG}^{3}$, HUIYUN ZHANG $^{4}$ and SHUJIAO LIU ${ }^{1}$ \\ ${ }^{1}$ Department of Geriatrics, Affiliated Hospital of Guilin Medical University, Guilin, Guangxi 541001; \\ ${ }^{2}$ Department of Cell Biology and Genetics, Guilin Medical University, Guilin, Guangxi 541005; ${ }^{3}$ Department of \\ Occupational and Environmental Health, School of Public Health, Guangxi Medical University, Nanning, Guangxi 530021; \\ ${ }^{4}$ Department of Endocrinology, Graduate School of Guilin Medical University, Guilin, Guangxi 541001, P.R. China
}

Received October 18, 2018; Accepted April 9, 2019

DOI: $10.3892 / \mathrm{mmr} .2019 .10252$

\begin{abstract}
Osteoporosis (OP) seriously affects the health and quality of life of elderly individuals and postmenopausal women, and the need to identify drugs that can prevent or treat OP remains urgent. Recently, several miRNAs have been reported to be involved in the differentiation of mesenchymal stem cells and osteoblasts; however, the role of miRNA (miR)-144 in regulating OP remains to be elucidated. In the present study, the expression levels of miR-144, secreted frizzled-related protein 1 (Sfrp1) and TNF- $\alpha$ in clinical samples were detected by the reverse transcription-quantitative polymerase chain reaction analysis and ELISA, respectively. 5-Ethynyl-2'-deoxyuridine staining, Hoechst 33258 staining, flow cytometry, a clone formation assay and Alizarin red staining were used to assess the effects of miR-144 combined with or without Sfrp1 small interfering RNA on the proliferation, apoptosis and osteoblastic differentiation of primary mesenchymal stem cells isolated from rats. Western blot assays were performed to assess the relevant mechanisms, and a dual luciferase reporter assay was used to detect the interaction between miR-144 and Sfrp1. The results showed that the levels of miR-144, Sfrp1 and TNF- $\alpha$ in clinical serum samples obtained from patients with postmenopausal OP were higher than those in serum samples obtained from postmenopausal women with normal bone density. There was a significant positive correlation between miR-144 and Sfrp1. Functional experiments demonstrated that miR-144 promoted proliferation, inhibited apoptosis and induced the osteoblastic
\end{abstract}

Correspondence to: Dr Shujiao Liu, Department of Geriatrics, Affiliated Hospital of Guilin Medical University, 15 Lequn Road, Guilin, Guangxi 541001, P.R. China

E-mail: 1sj1817@163.com

Key words: microRNA-144, proliferation, differentiation, bone mesenchymal stem cells, secreted frizzled-related protein 1 differentiation of bone marrow-derived mesenchymal stem cells by targeting Sfrpl. It was also shown that miR-144 may help regulate OP by activating the $\mathrm{Wnt} / \beta$-catenin pathway. These data suggest miR-144 as a novel target for preventing and treating OP.

\section{Introduction}

Osteoporosis (OP) is a systemic bone metabolic disorder characterized by reduced bone density and the degeneration of bone microstructure, which results in increased bone brittleness and fracture risk $(1,2)$. As one of the most common bone metabolic disorders, OP adversely affects the health and quality of life of elderly individuals and postmenopausal women $(3,4)$. Numerous studies have sought to identify genes associated with OP and sensitive genetic markers, and then use the information to predict the occurrence and development of OP.

Several hormones, cell types and humoral factors regulate the bone reconstruction process by promoting or inhibiting the development of osteoblasts and osteoclasts during the remodeling/modeling process (5-7). Bone marrow-derived mesenchymal stem cells (BMSCs) are the source osteoblasts in bone tissue, and affect the numbers and functional activities of osteoblasts (8). BMSCs also serve a major role in bone reconstruction and help maintain a balance between bone formation and bone resorption $(9,10)$. A previous study reported that BMSCs can regulate the occurrence and development of postmenopausal OP (11). The most fundamental reason for abnormal bone reconstruction in cases of postmenopausal OP is functional defects of BMSCs, including decreased proliferative activity and adipogenic differentiation ability, and increased lipid differentiation ability $(12,13)$. Therefore, BMSCs were selected as target cells in which to investigate the occurrence and development of OP.

MicroRNAs (miRNAs) are endogenous, non-coding small RNAs composed of 21-23 nucleotides (14), which have been reported to be involved in numerous complex cellular processes, including cell proliferation, differentiation, division, apoptosis and gene regulation $(15,16)$. In recent 
years, miRNAs have been shown to be involved in the occurrence, development and prognosis of various diseases, and have also been used to diagnose and treat diseases $(17,18)$. miRNAs also regulate the differentiation of mesenchymal stem cells and osteoblasts $(19,20)$. During the processes of bone differentiation and regeneration, abnormal miRNA expression is closely associated with OP $(21,22)$. For example, the miR-34c-induced silencing of $M C 3 T 3-E 1$ reduces the inhibitory effect of vaspin on osteoblast differentiation (23); furthermore, miRNA (miR)-433-3p can regulate osteoblastic differentiation by targeting Dickkopf-1 (DKK1) (24). As an important miRNA, miR-144 is known to be involved in the occurrence and development of various human diseases, including ovarian cancer (25), cervical cancer (26) and anaplastic thyroid carcinoma (27). For example, miR-144 acts as a tumor suppressor in osteosarcoma by inhibiting cell proliferation and inducing apoptosis (28). miR-144 also aggravates intestinal hyperpermeability and destroys the epithelial barrier (29). Reports have demonstrated that mammalian target of rapamycin, occludin and zonula occludens 1 are all target genes of miR-144 (29,30). However, the effect of miR-144 on the occurrence and development of OP and the associated molecular mechanisms have not been reported.

The regulation of osteoblast differentiation is a complex process involving multiple genes and pathways, including the Notch signaling pathway, bone morphogenetic protein (BMP)/Smad signaling pathway and Wnt signaling pathway (31-33). The $\mathrm{Wnt} / \beta$-catenin signaling pathway serves a vital role in regulating osteoblastic differentiation and osteogenic matrix formation $(34,35)$; therefore, the use of Wnt/ $\beta$-catenin pathway antagonists represents a novel approach for treating OP. As a family of secretory glycoproteins, secreted frizzled-related proteins (SFRPs) are a class of extracellular antagonists of the Wnt signaling pathway (36). A previous study demonstrated that SFRPs can antagonize the Wnt signaling pathway by competitively binding to the Frizzled receptor in a cysteine-rich domain (37). SFRP1 is one of the most important proteins in the SFRPs family and a negative regulator of human osteoblast and osteocyte survival (37). However, whether SFPR1 is involved in the process by which miR-144 regulates OP remains to be elucidated.

In the present study, the expression of miR-144 and its target gene SFPR1 were detected in clinical serum samples obtained from patients with postmenopausal OP and in postmenopausal women with normal bone density. Subsequently, primary bone mesenchymal stem cells (BMSCs) were isolated from rats, and flow cytometry and EdU and Hoechst 33258 staining methods were used to detect cell apoptosis. Following completion of an osteoblast induction culture, a clone formation assay was used to assess changes in the ability of cells to form clones, and Alizarin red staining was used to determine the extent to which the BMSCs had differentiated into osteoblasts. The mechanism by which miR-144 regulates OP was also investigated. The results not only provide a basis for investigating the mechanism by which miR-144 is involved in the occurrence and development of OP, but also suggest a novel molecular target for preventing and treating OP.

\section{Materials and methods}

Collection of clinical samples. The collection and analysis of all clinical samples was approved by the Ethics Committee of the Affiliated Hospital of Guilin Medical University (Guilin, China; GLMUIA2016002). In total, 15 pairs of serum samples were collected from patients with postmenopausal OP and postmenopausal women with a normal bone mineral density. The samples were collected from January 2016 to May 2018 at the Affiliated Hospital of Guilin Medical University. The donors, including the postmenopausal women with OP and women with normal bone mineral density had no other diseases, and ranged in age between 54 and 64 years.

Isolation and culture of BMSCs. The gradient centrifugation method was used to isolate MSCs from the bone marrow, which had been collected from Sprague-Dawley (SD) rats. A total of 4 male SD rats (8 weeks old; $200 \pm 20 \mathrm{~g}$ ) were specific pathogen-free (certificate no. SCXK2011-0015), purchased from the Animal Center of Southern Medical University (Guangzhou, China) and housed in an animal experimental center. The rats were housed under standard conditions ( $22 \pm 2^{\circ} \mathrm{C} ; 40-60 \%$ relative humidity; 12:12-h light/dark cycle). Throughout the rearing period, the rats were provided access to food and water ad libitum. The protocols for all animal experiments were approved by the Animal Ethics Committee of the Affiliated Hospital of Guilin Medical University. The BM aspirates were cultured in MEM-a medium (Thermo Fisher Scientific, Inc., Waltham, MA, USA) containing 10\% FBS (Gibco; Thermo Fisher Scientific, Inc.). Cells were cultured in a humidity incubator at $37^{\circ} \mathrm{C}$ with $5 \% \mathrm{CO}_{2}$. Following culture for $24 \mathrm{~h}$, the suspended cells were removed and the adherent cells were cleaned with phosphate-buffered saline (PBS) solution. The adherent cells were then cultured for $\sim 10$ days with two changes of medium (PBS). When the cells reached $\sim 75 \%$ confluence, they were harvested for use in experiments.

Transfection. To achieve changes in miR-144 expression, BMSCs $\left(2 \times 10^{4}\right.$ cells $\left./ \mathrm{ml}, 200 \mu \mathrm{l}\right)$ were seeded in 48 -well plates and transfected with miR-144 mimic $(50 \mathrm{nmol} / \mathrm{l})$ or inhibitor (50 nmol/1; Guangzhou Ribobio Co., Ltd., Guangzhou, China) using Lipofectamine ${ }^{\circledR} 2000$ (Invitrogen; Thermo Fisher Scientific, Inc.) as a transfection reagent, according to the manufacturer's instructions. Following incubation for $6 \mathrm{~h}$, cells were cultured in MEM-a complete medium for $48 \mathrm{~h}$. The miR-144 mimic and miR-144 inhibitor were used to achieve the overexpression and knockdown of miR-144, respectively. BMSCs treated with Lipofectamine 2000 reagent alone (Mock) were used as control cells. The sequences of the miR-144 mimic, miR nonsense strand negative control (NC) and miR-144 inhibitor were as follows: Mimic, sense 5'-UAC AGUAUAGAUGAUGUACU-3'; miR NC strand, sense 5'-UUCUCCGAACGUGUCACGUTT-3'; inhibitor, sense 5'-AGUACAUCAUCUAUACUGUA-3'. To achieve changes in the expression of Sfrp1, the BMSCs were treated with Sfrp1 small interfering (si)RNA (5 pmol/1) (three Sfrp1 siRNAs purchased from Sangon Biotech Co., Ltd., Shanghai, China) or a nonsense strand NC. Following incubation for $6 \mathrm{~h}$, cells were cultured in MEM-a complete medium for $48 \mathrm{~h}$. The sequences of the SFRP1 siRNAs and control siRNA were 
as follows: NC of siRNA, sense 5'-UUCUCCGAACGUGUC ACGUTT-3'; siRNA-1 (789-811) sense 5'-GCCACAACU UCCUCAUCAUUU-3'; siRNA-2 (561-583) sense 5'-CGU GUGACAACGAGUUGAAUU-3'; siRNA-3 (836-858) sense 5'-CUCACAGCCAUUCACAAGUUU-3'. The sfrp1 siRNAs were used to knock down the expression of Sfrpl. siRNA-2 (siSfrp1-2) was selected for use in subsequent knockdown experiments following initial characterization of the three siRNAs. Reverse transcription-quantitative polymerase chain reaction (RT-qPCR) and western blot methods were used to detect transfection efficiency.

Dual luciferase reporter assay. The binding site for miR-144 in Sfrp1 mRNA was predicted by using TargetScan release 7.2 (http://www.targetscan.org/vert_72/), and the results indicated that Sfrpl was a target gene for miR-144. To confirm that Sfrpl is a target gene for miR-144, 293T cells $\left(2 \times 10^{4}\right.$ cells $/ \mathrm{ml}$, $200 \mu$; ; American Type Culture Collection, Manassas, VA, USA) were seeded in 48-well plates and transfected with 400 ng wild-type Sfrp1 3'-UTR (WT-Sfrp1) or mutant Sfrp1 3'-UTR (MUT-Sfrp1) cloned into the psiCHECK2 plasmid (Promega Corporation, Madison, WI, USA) together with $50 \mathrm{nmol} / 1 \mathrm{miR}-144$ nonsense strand or mimic using Lipofectamine 2000. Following incubation for 6 h, 293T cells were cultured in DMEM complete medium for $48 \mathrm{~h}$, and then luciferase activity was detected with a dual-luciferase reporter assay (Promega Corporation). The relative luciferase activity was calculated using firefly luciferase activity as an internal control.

$R T-q P C R$ analysis. The expression levels of miR-144, Sfrp1 and Runt-related transcription factor 2 (Runx2) were detected by RT-qPCR analysis. Total RNA was extracted from serum with a GenElute ${ }^{\mathrm{TM}}$ Plasma/Serum RNA Purification Mini kit (Sigma-Aldrich; Merck KGaA, Darmstadt, Germany) according to the manufacturer's instructions, and then transcribed into cDNA with the use of a Bestar qPCR RT kit (DBI Bioscience, Ludwigshafen, Germany). RT was conducted as follows: $37^{\circ} \mathrm{C}$ for $15 \mathrm{~min}$ and $98^{\circ} \mathrm{C}$ for $5 \mathrm{~min}$. qPCR was performed using a Bestar ${ }^{\mathrm{TM}}$ qPCR Master Mix (DBI Bioscience) under the following conditions: $95^{\circ} \mathrm{C}$ for $2 \mathrm{~min}$, followed by 40 cycles of $94^{\circ} \mathrm{C}$ for $20 \mathrm{sec}, 58^{\circ} \mathrm{C}$ for $20 \mathrm{sec}$ and $72^{\circ} \mathrm{C}$ for $20 \mathrm{sec}$, and finally extension at $72^{\circ} \mathrm{C}$ for $4 \mathrm{~min}$. The RT-qPCR analysis was performed on an Agilent Stratagene Mx3000P Sequence Detection system (Agilent Technologies, Inc., Santa Clara, CA, USA) using the following primer sequences: GAPDH, forward 5'-CCTCGTCTCATAGAC AAGATGGT-3' and reverse 5'-GGGTAGAGTCATACTGGA ACATG-3'; miR-144, forward 5'-ACACTCCAGCTGGGT ACAGTATAGATGATG-3' and reverse 5'-CTCAACTGG TGTCGTGGAGTCGGCAATTCAGTTGAGAGTACATC-3'; U6, forward 5'-CTCGCTTCGGCAGCACA-3' and reverse 5'-AACGCTTCACGAATTTGCGT-3'; Sfrp1, forward 5'-TCA TGCAGTTCTTCGGCTTC-3' and reverse 5'-TTCAACTCG TTGTCACACGG-3'; Runx2, forward 5'-CATTCGCCTCAC AAACAACC-3' and reverse 5'-AGAAGTTTTGCTGACACG GT-3'. GAPDH and U6 were used as the control for the relative quantification of mRNA or miRNA, respectively. The relative levels of gene expression were calculated using the $2^{-\Delta \Delta C q}$ method (38).
ELISA. Blood samples from patients with postmenopausal OP and postmenopausal women with normal bone mineral density were collected in serum separator tubes (BD Biosciences, San Jose, CA, USA), clotted for $15 \mathrm{~min}$ and then centrifuged at $3,000 \mathrm{x}$ g for $5 \mathrm{~min}$ at $4^{\circ} \mathrm{C}$. Serum were collected and stored at $-80^{\circ} \mathrm{C}$ until detected. The serum levels of Sfrp1 and TNF- $\alpha$ in patients with postmenopausal OP and postmenopausal women with normal bone mineral density were determined by ELISA, according to the manufacturer's instructions. The testing kits for Sfrp1 (cat. no. CSB-E15074h) and TNF- $\alpha$ (cat. no. CSB-E04740h) were purchased from Cusabio Biotech Co., Ltd. (Wuhan, China). The absorbance was measured at $450 \mathrm{~nm}$ using the SpectraMax M5 microplate reader (Molecular Devices, LLC, Sunnyvale, CA, USA).

5-Ethynyl-2'-deoxyuridine (EdU) staining. Thymidine analog EdU staining was used to detect cell proliferation. The MSCs $\left(1 \times 10^{4} /\right.$ well) were seeded in 96-well plates (Corning Inc., Corning, NY, USA) and cultured in a humidity incubator at $37^{\circ} \mathrm{C}$ with $5 \% \mathrm{CO} 2$. In atmosphere. The MSCs wee transfected as previously mentioned and then treated with $50 \mu \mathrm{M}$ EdU (cat. no. C10310-2, Guangzhou Ribobio Co., Ltd.) for $2 \mathrm{~h}$, following which, they were collected and stained with reagents in the Apollo ${ }^{\circledR} 643$ EdU labeling kit (cat. no. C10310-2, Guangzhou Ribobio Co. Ltd.). The cell nuclei were stained with Hoechst 33342 (cat. no. C1022, Beyotime Institute of Biotechnology; Haimen, China). The stained cells were then observed and images were captured with a fluorescence confocal microscope (Olympus FV1000, Olympus Corporation, Tokyo, Japan).

Apoptosis assay. Flow cytometry and a Hoechst staining assay were used to determine the effect of miR-144 on apoptosis. The BMSCs $\left(1 \times 10^{6}\right)$ were seeded into 6-well plates (Corning Inc.), and then transfected with miR-144 mock, miR-144 mimic or miR-144 inhibitor plasmids, respectively. After $48 \mathrm{~h}$, the cells were collected and stained with reagents in an Annexin V-FITC/PI apoptosis detection kit (Sigma-Aldrich; Merck $\mathrm{KGaA}$ ) at room temperature for $10 \mathrm{~min}$ in the dark, following which, they were immediately counted with a BD FACSCalibur flow cytometry (BD Biosciences). CellQuest software (version 3.3; BD Biosciences) was used to assess the resultant data. Apoptosis-induced chromatin pycnosis in the nucleus was detected by Hoechst staining. The transfected cells were seeded into 24 -well plates and cultured for $48 \mathrm{~h}$, following which, they were fixed in $4 \%$ (w/v) paraformaldehyde at $4^{\circ} \mathrm{C}$ for $20 \mathrm{~min}$. The cells were then stained with reagents in a Hoechst 33258 staining kit (cat. no. C1011, Beyotime Institute of Biotechnology) at room temperature for $30 \mathrm{~min}$ in the dark, and images were captured with a laser scanning confocal microscope (LSM700; Zeiss AG, Oberkochen, Germany).

Colony formation assay. Briefly, the BMSCs transfected with plasmids were seeded into 6-well plates (Corning) and maintained in a medium containing $10 \%$ FBS; the medium was refreshed every 2 days. Following 7 days of incubation, the cells were stained with $0.1 \%$ crystal violet solution (Sigma-Aldrich; Merck KGaA) at room temperature for $10 \mathrm{~min}$, and the numbers of colonies containing $>50$ cells were 
counted. Colonies were observed using a microscope imaging system (Nikon Corporation, Tokyo, Japan).

Western blot analysis. RIPA buffer (Sigma-Aldrich; Merck $\mathrm{KGaA}$ ) reconstituted with a proteasome inhibitor (Roche Diagnostics, Indianapolis, IN, USA) and phosphatase inhibitors (Roche Diagnostics) was used to lyse cells. The concentration of samples were determined using a Pierce ${ }^{\mathrm{TM}}$ BCA Protein Assay Kit (Pierce; Thermo Fisher Scientific, Inc.). Samples of total cellular protein were loaded onto 5-10\% SDS-polyacrylamide gels ( $20 \mu \mathrm{g}$ of protein per lane) and then separated by electrophoresis. The separated protein bands were transferred onto PVDF membranes (EMD Millipore, Billerica, MA, USA), then the PVDF membranes were blocked with $5 \%$ bovine serum albumin solution (Sigma-Aldrich; Merck KGaA) for $1 \mathrm{~h}$ at room temperature, and then incubated with antibodies against Runx2 (1:1,000; cat. no. 8486, CST Biological Reagents Co., Ltd.), cyclin-dependent kinase (CDK)4 (1:3,000; cat. no. 12790, CST Biological Reagents Co., Ltd.), Sfrp1 (1:4,000; cat. no. 3534, CST Biological Reagents Co., Ltd.), Wnt1 (1:1,000; cat. no. ab85060, Abcam), $\beta$-catenin (1:5,000; cat. no. 8480, CST Biological Reagents Co., Ltd.) or GAPDH (1:10,000; cat. no. ab8245, Abcam) overnight at $4^{\circ} \mathrm{C}$. HRP-conjugated goat anti-rabbit (cat. no. BA1054) or anti-mouse (cat. no. BA1050) antibodies (1:20,000; Boster Biological Technology, Pleasanton, CA, USA) were used as secondary antibodies; membranes were incubated with secondary antibodies for $2 \mathrm{~h}$ at room temperature. An enhanced chemiluminescence kit (GE Healthcare, Chicago, IL, USA) was used to visualize protein bands.

Immunofluorescence assay. The effects of miR-144 on the expression of Sfrp1 and Runx2 were investigated by immunofluorescence. Briefly, the BMSCs were fixed and permeabilized with $4 \%(\mathrm{w} / \mathrm{v})$ paraformaldehyde at $4^{\circ} \mathrm{C}$ for $20 \mathrm{~min}$. The cells were then incubated overnight at $4^{\circ} \mathrm{C}$ with primary antibodies (anti-Sfrp1 or anti-Runx2, 1:100 dilution), following which they were washed and stained with either FITC-conjugated goat anti-mouse IgG (1:100; cat. no. sc-2010, Santa Cruz Biotechnology, Inc., Dallas, TX, USA) or phycoerythrin-conjugated IgG (1:1,000; cat. no. sc-3738, Santa Cruz Biotechnology, Inc.) at $4^{\circ} \mathrm{C}$ for $30 \mathrm{~min}$ in the dark. The cell nuclei were stained with DAPI $(10 \mu \mathrm{g} / \mathrm{ml})$ at room temperature for $5 \mathrm{~min}$ in the dark. A laser scanning confocal microscope (LSM700; Zeiss AG) was used to capture the images.

Alizarin red staining. To induce osteoblast differentiation, the BMSCs were cultured in complete medium for the osteogenic differentiation of rat BMSCs (Cyagen Biosciences, Inc., Santa Clara, CA, USA), according to the manufacturer's guidelines. After 3 weeks of culture, calcium nodi, which are indicative of osteogenic differentiation, were detected by Alizarin red staining. The BMSCs were then transferred onto slides and fixed in $10 \%$ ethanol, following which the slides were incubated in $0.1 \%$ Alizarin red staining solution (cat. no. G8550, Beijing Solarbio Science \& Technology Co., Ltd., Beijing, China) at room temperature for $30 \mathrm{~min}$ and then rinsed with distilled water. Following a final wash, images of the stained cells were captured using a microscope imaging system (Nikon Corporation).
Alkaline phosphatase (ALP) assay. The activity of ALP, a marker of early osteoblastic differentiation, was detected and used to demonstrate osteogenic differentiation. ALP activity assays were performed following 14 days of osteogenic differentiation. Briefly, the cells were collected and lysed with lysis buffer, and the total protein concentration was analyzed with a bicinchoninic acid protein assay kit (Pierce; Thermo Fisher Scientific, Inc.). ALP activity was determined using a phosphatase assay kit (Beyotime Institute of Biotechnology), with the absorbance measured at $405 \mathrm{~nm}$.

Reactive oxygen species (ROS) assay. Flow cytometric assays were preformed to detect the levels of ROS in BMSCs. The BMSCs $\left(1 \times 10^{6}\right)$ were seeded into 6-well plates and cultured for $24 \mathrm{~h}$. The BMSCs were collected and stained with the reagents of a Reactive Oxygen Species Assay kit (cat. no. MAK144, Sigma-Aldrich; Merck KGaA) at $37^{\circ} \mathrm{C}$ for $1 \mathrm{~h}$. Following staining, the cells were immediately counted with a BD FACS Calibur flow cytometry (BD Biosciences). CellQuest Pro software was used to analyze the data.

Statistical analysis. Each experiment was repeated at least three times, and results are expressed as the mean \pm SEM. Student's t-test and one-way ANOVA with the Newman-Keuls post hoc test were used to analyze the data. All statistical was analyses were performed using GraphPad Prism Version 7.0 software (GraphPad Software, Inc., La Jolla, CA, USA). $\mathrm{P}<0.05$ was considered to indicate a statistically significant difference.

\section{Results}

Expression of miR-144, Sfrpl and TNF- $\alpha$ in clinical samples. In order to investigate the biological roles of miR-144, Sfrp1 and TNF- $\alpha$ in the development of OP, their respective expression levels were compared clinically in patients with postmenopausal OP $(n=15)$ and postmenopausal women with a normal bone density $(n=15)$. The RT-qPCR results showed that the expression of miR-144 was upregulated in the patients with OP compared with its expression in the normal group (Fig. 1A). The serum levels of Sfrp1 and TNF- $\alpha$ were detected by ELISA, which showed trends that were similar to that of miR-144 (Fig. 1B and C). In addition, correlation analysis $(n=30)$ revealed a significant correlation between the serum levels of miR-144 and Sfrp1 (Fig. 1D). These results indicated that miR-144 and Sfrp1 are involved in the development of OP.

Effect of miR-144 on BMSC proliferation and apoptosis. To examine the role of miR-144 in the function of BMSCs, BMSCs were first isolated from rats and then transfected with miR-144 mimic or the miR-144 inhibitor. EdU staining was used to evaluate the effect of miR-144 on BMSC proliferation. The results showed that overexpression of miR-144 efficiently promoted the proliferation of BMSCs when compared with BMSCs in the mock groups (Fig. 2A and B), whereas the opposite effect was observed for BMSCs in the miR-144 inhibitor group (Fig. 2A and B). In addition, Hoechst 33258 staining was used to detect the effect of miR-144 on the nuclear morphological features of BMSCs. As shown in 

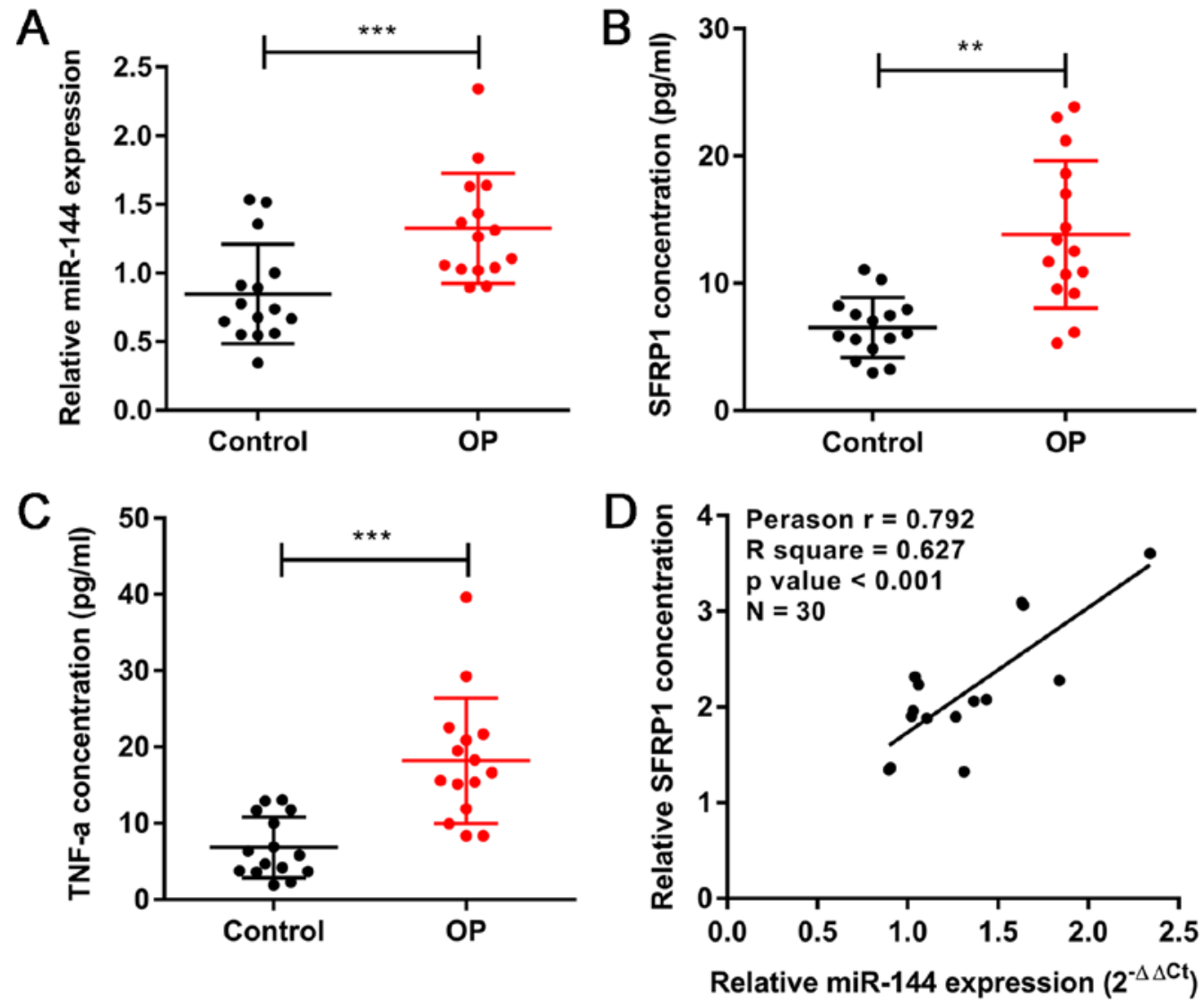

Figure 1. Expression of miR-144, Sfrp1 and TNF- $\alpha$ in clinical samples. (A) Expression of miR-144 was detected by reverse transcription-quantitative polymerase chain reaction analysis. Serum levels of (B) Sfrp1 and (C) TNF- $\alpha$ were detected by ELISA. (D) Correlation analysis was performed to examine the relationship between miR-144 and Sfrp1. Data represent the mean \pm SEM. ${ }^{* *} \mathrm{P}<0.01$ and ${ }^{* * * *} \mathrm{P}<0.001$. OP, osteoporosis; miR, microRNA; Sfrp1, secreted frizzled-related protein 1.

Fig. 2C and D, the number of BMSCs with chromatin condensation was markedly increased following treatment with the miR-144 inhibitor when compared with the BMSCs in the mock group. However, there was no change in the BMSCs treated with the miR-144 mimic (Fig. 2C and D). As shown in Fig. $2 \mathrm{~B}$ and $\mathrm{C}$, the ratio of positive cells indicates the ratio of positive to total cells. An apoptosis detection kit was then used to detect the role of miR-144 in BMSC apoptosis. The results showed that the miR-144 inhibitor significantly increased the numbers of apoptotic BMSCs when compared with the BMSCs in the mock group, whereas there was no difference between the BMSCs treated with the miR-144 mimic and the mock group (Fig. 2E and F). It was also found that the absence of miR-144 significantly increased the levels of intracellular ROS in BMSCs compared with those in BMSCs in the mock groups (Fig. 2G and $\mathrm{H}$ ), indicating that the absence of miR-144 may induce apoptosis in BMSCs by increasing their concentrations of reactive oxygen species.

miR-144 promotes the proliferation and differentiation of BMSCs. To further examine the effect of miR-144 on the proliferation of BMSCs, a colony formation assay was used to evaluate the effect of miR-144 on BMSC proliferation. The results indicated that the overexpression of miR-144 efficiently promoted the proliferation of BMSCs, whereas miR-144 knockdown efficiently inhibited BMSC proliferation compared with that in the mock group (Fig. 3A). Subsequently,
Alizarin red staining was performed to detect the numbers of calcium nodi in the BMSCs. The results showed that the overexpression of miR-144 significantly increased the numbers of calcium nodi, whereas miR-144 knockdown efficiently decreased the numbers of calcium nodi in the BMSCs, compared with the numbers in the corresponding mock groups (Fig. 3B), indicating that miR-144 may induce the osteoblastic differentiation of BMSCs in vitro. The ALP activity assay was used to further examine the role of miR-144 in the osteogenic differentiation of BMSCs. The results showed that the overexpression of miR-144 significantly increased ALP activity, whereas miR-144 knockdown decreased ALP activity (Fig. 3C). When taken together, these results indicate that miR-144 can induce the osteoblastic differentiation of BMSCs in vitro.

Expression of Sfrpl and Runx2 in BMSCs is regulated by $m i R$-144. It is generally known that miRNA binds to the 3 '-UTR of its target mRNAs and thereby regulates gene expression. The TargetScan website (http://www.targetscan.org/) was used to predict the binding site for miR-144, and the results indicated that Sfrpl was a potential target of miR-144. To investigate the role of miR-144 in the expression of Sfrpl, BMSCs were transfected with miR-144 mimic or inhibitor and the expression levels of Sfrp1, miR-144 and Runx2 were detected by RT-qPCR and western blot methods. As shown in Fig. 4, the expression levels of miR-144 in the miR-144 mimic group and 
A
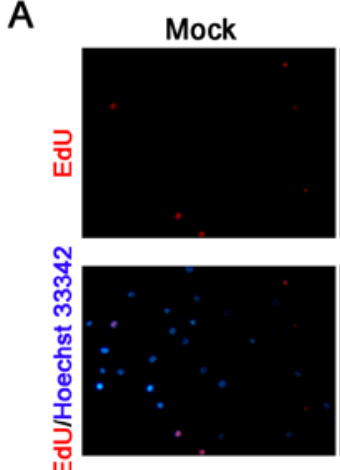

C

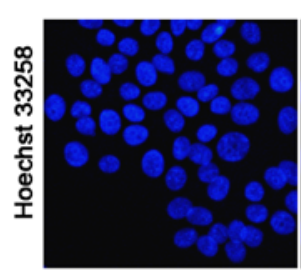

E

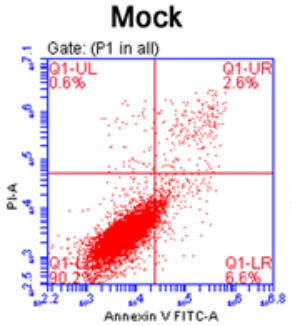

G

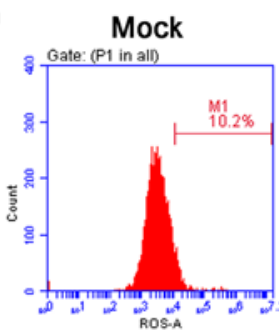

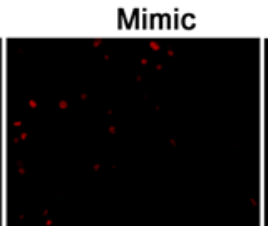
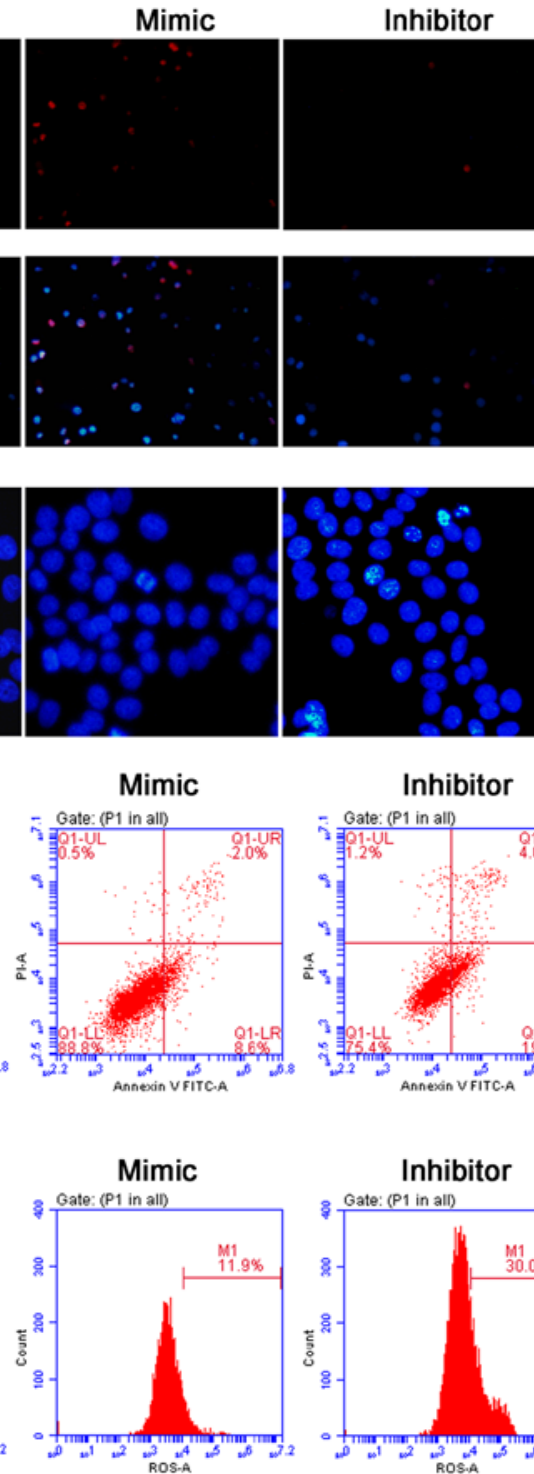
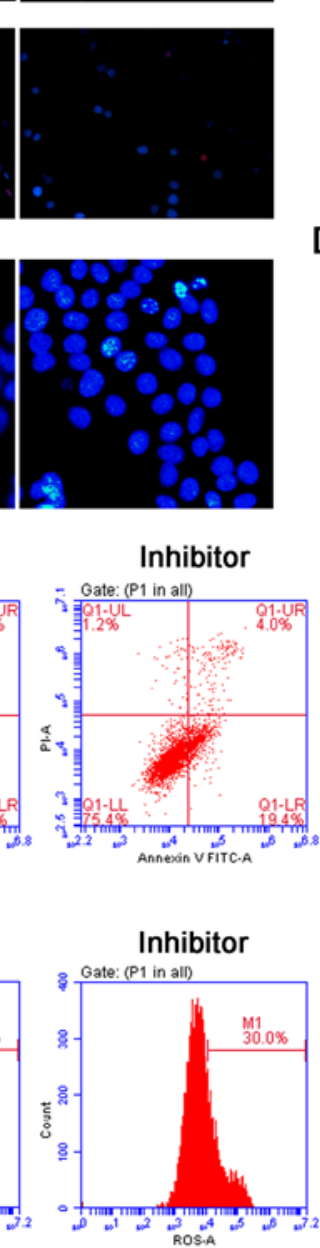

B
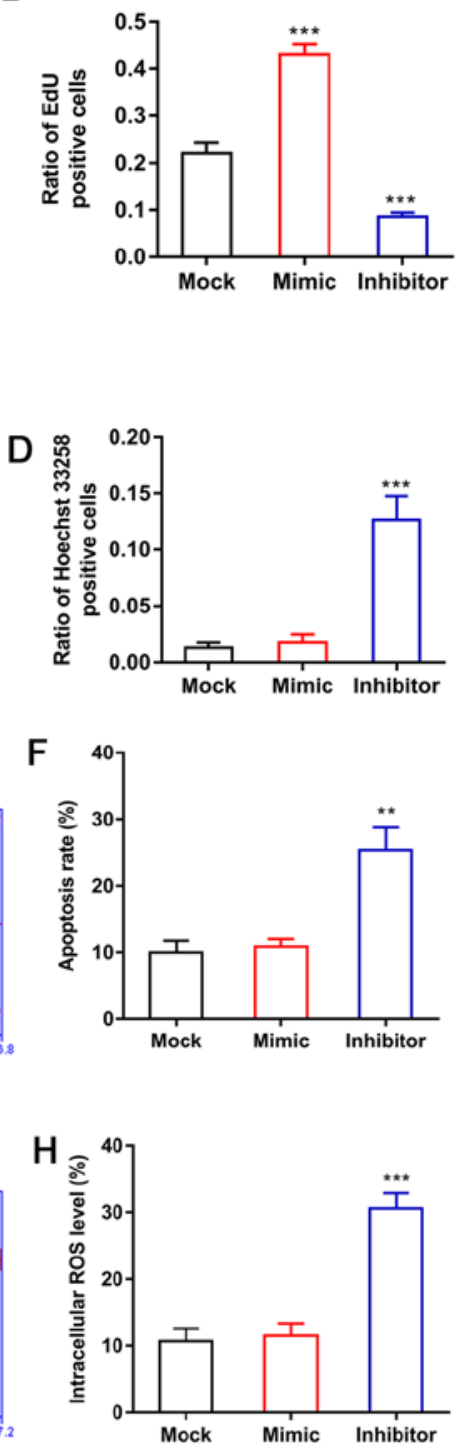

Figure 2. Effect of miR-144 on BMSC proliferation and apoptosis. (A) EdU staining was used to evaluate and the effect of miR-144 on BMSC proliferation (magnification, x100). (B) Ratio of Edu-positive cells to total cells. (C) Effect of miR-144 on the nuclear morphological features of BMSCs was observed following Hoechst 33258 staining (magnification, x200). (D) Ratio of Hoechst 33258-positive cells to total cells. (E) Effect of miR-144 on BMSC apoptosis was detected by flow cytometry and (F) quantified. (G) Intracellular ROS in BMSCs were detected by flow cytometry and (H) quantified. The group of BMSCs transfected with miR-144 mimic or the miR-144 inhibitor were compared with BMSCs in the mock groups. Data represent the mean \pm SEM. ${ }^{* *} \mathrm{P}<0.01$ and ${ }^{* * *} \mathrm{P}<0.001$. miR, microRNA; BMSCs, bone marrow-derived mesenchymal stem cells; ROS, reactive oxygen species.

miR-144 inhibitor group were significantly upregulated and downregulated, respectively, compared with their levels in the corresponding mock groups (Fig. 4A). Furthermore, the mRNA and protein levels of Sfrp1 in the BMSCs transfected with miR-144 mimic or miR-144 inhibitor were significantly reduced and increased, respectively (Fig. 4A and B). The immunofluorescence assay showed similar results (Fig. 4C). The present study also investigated how the mRNA and protein levels of Runx 2 were altered by miR-144, and found that Runx2 levels in the BMSCs were significantly increased when miR-144 was overexpressed (Fig. 4A and B). By contrast, the expression of Runx2 in BMSCs was significantly reduced when miR-144 was knocked down (Fig. 4A and B), and the immunofluorescence assays showed similar results (Fig. 4C). The protein levels of CDK4, Wnt 1 and $\beta$-catenin were also markedly increased by miR-144 (Fig. 4B).
miR-144 promotes the proliferation and differentiation of BMSCs by downregulating the expression of Sfrpl. The binding site for miR-144 in Sfrp1 mRNA was predicted using the TargetScan website, and Sfrpl was suggested as a target gene for miR-144 in BMSCs. The luciferase reporter assay was used to examine the direct interaction between miR-144 and Sfrp1. The 3'-UTR sequences of the WT and MUT Srfp1 were sequenced, and the results revealed that the MUT Sfrp1 3'-UTR gene had successfully mutated in the target region (data not shown). The 3'-UTR sequences of the WT and MUT Srfp1 were cloned into a psiCHECK2 plasmid in which the firefly luciferase gene is constitutively expressed. The results showed that overexpression of miR-144 significantly decreased the luciferase activity of the WT Sfrp1 plasmid, compared with that of the other three groups (Fig. 5A). By contrast, there was no effect on the luciferase activity of the MUT Sfrp1 
A
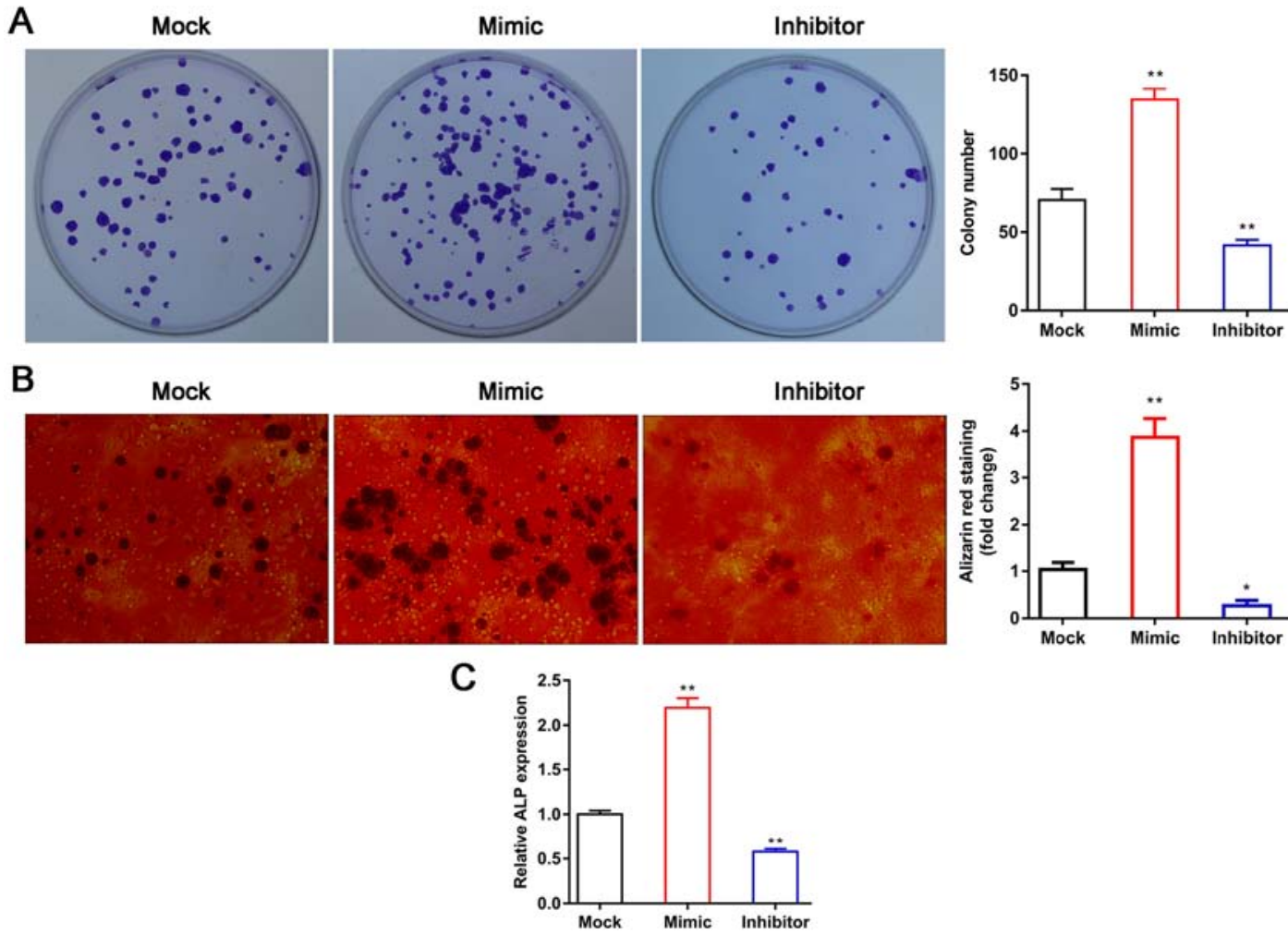

Figure 3. miR-144 promotes the proliferation and differentiation of BMSCs. (A) Effect of miR-144 on BMSC proliferation was evaluated with the colony formation assay. (B) Alizarin red staining was performed following 3 weeks of osteogenic differentiation to detect the calcium nodi in BMSCs (magnification, $\mathrm{x} 200$ ). (C) ALP activity assays were performed following 14 days of osteogenic differentiation. Data represent the mean $\pm \mathrm{SEM}$. ${ }^{*} \mathrm{P}<0.05$ and ${ }^{* *} \mathrm{P}<0.01$. miR, microRNA; BMSCs, bone marrow-derived mesenchymal stem cells; ALP, alkaline phosphatase.

plasmid compared with that in the NC group (Fig. 5A). Taken together, these results indicate that miR-144 can bind to the 3'-UTR of Sfrp1 mRNA and thereby regulate the expression of Sfrpl. To further examine the effects of Sfrp1 and miR-144 on the function of BMSCs, BMSCs were transfected with Sfrp1 siRNA. The mRNA and protein levels of Sfrp1 were significantly reduced by Sfrp1 siRNA, particularly in the second Sfrp1 siRNA group, compared with that in the NC group (Fig. 5B and C). In follow-up experiments, the second Sfrp1 siRNA was used to interfere with the expression of Sfrpl. The colony formation assay, Alizarin red staining and ALP activity assay were used to further examine how Sfrp1 may influence miR-144 in promoting the proliferation and differentiation of BMSCs. The results showed that Sfrp1 suppressed the proliferation of BMSCs compared with that of the group of BMSCs co-transfected with the miR-144 inhibitor and NC siRNA (Fig. 5D and F), and also decreased calcium nodus formation (Fig. 5E and G) and ALP activity (Fig. 5H). In addition, the protein levels of Runx $2, \mathrm{CDK} 4$, Wnt 1 and $\beta$-catenin were markedly increased in the BMSCs co-transfected with the miR-144 inhibitor and Sfrp1 siRNA compared with the those in the group of BMSCs co-transfected with the miR-144 inhibitor and NC siRNA (Fig. 5I). It is well known that the Wnt/ $\beta$-catenin pathway serves an important role in cell proliferation and differentiation, and that Wnt signaling can be downregulated by Sfrp1 (39). It is also known that Runx2 is a key transcription factor associated with osteoblastic differentiation. When combined with our previous studies, these results suggest that miR-144 promotes the proliferation and differentiation of BMSCs by upregulating the expression of Runx2, CDK4, Wnt1 and $\beta$-catenin and downregulating the expression of Sfrp1.

\section{Discussion}

OP is a common disease among older adults and postmenopausal women $(1,3)$. The degeneration of osteoblasts, in terms of their function and quantity, aggravates the occurrence and development of OP; however, BMSCs can continue to differentiate into osteoblasts following directed induction, which has become an important method for preventing and curing OP $(10,40)$. The numbers and functions of BMSCs are key factors involved in maintaining the normal physiological function of bones, and the osteogenic capacity of BMSCs in bone marrow decreases when OP occurs (8-10). Therefore, investigations on how BMSCs differentiate into osteoblasts have become a primary focus in the search for novel treatments for OP. A recent study showed that miRNAs may be involved in cell proliferation and osteoblastic differentiation (41). The present study confirmed that the expression of miR-144 was markedly increased in clinical samples from patients with OP. Furthermore, miR-144 silencing inhibited the proliferation and promoted the apoptosis of BMSCs, suggesting that miR-144 may have a key regulatory role in the progression of OP. In addition, the numbers of calcium nodi in BMSCs was significantly increased following treatment with miR-144 mimic, indicating that miR-144 induced the osteoblastic differentiation of BMSCs. 

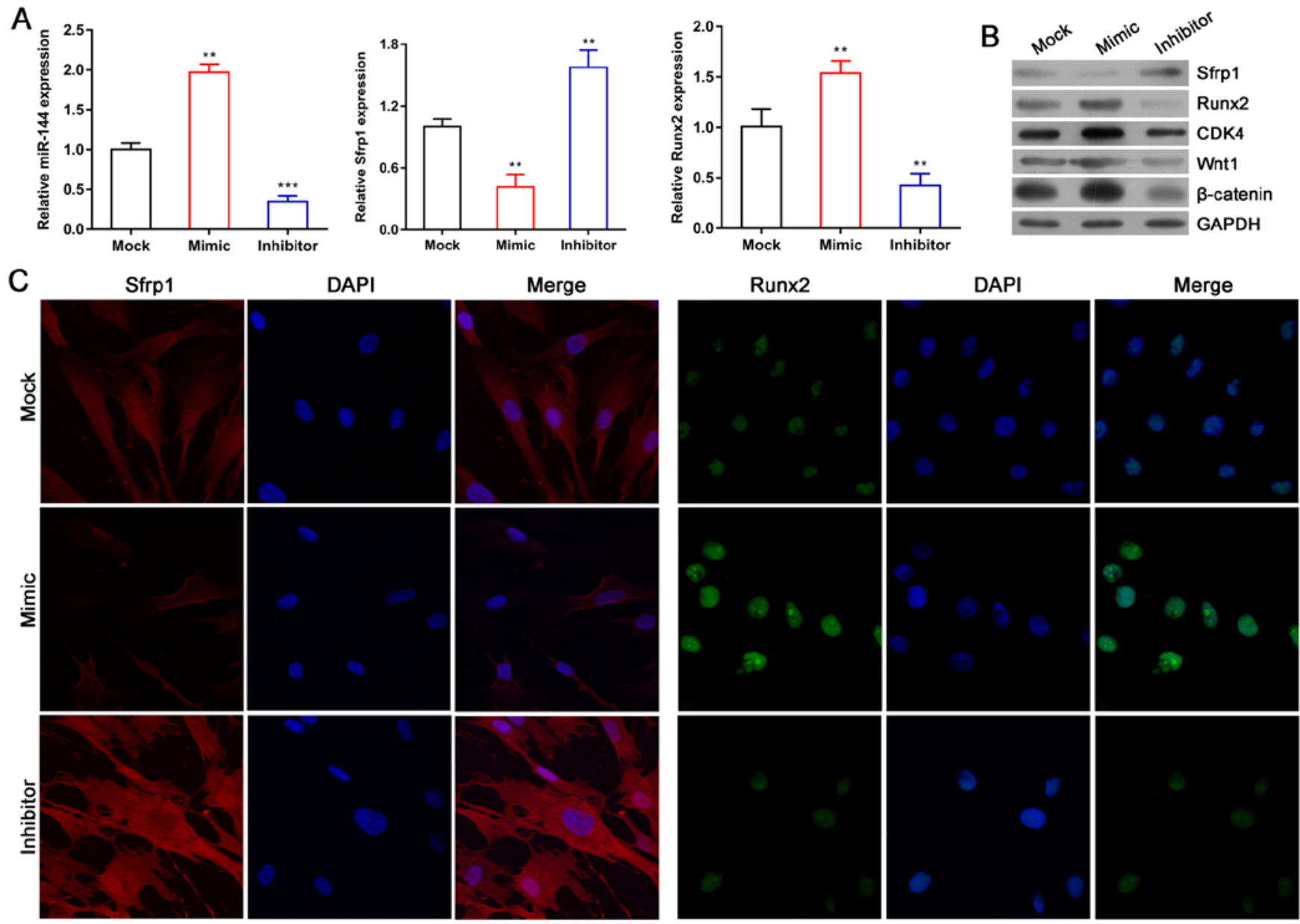

Figure 4. Expression of Sfrp1 and Runx2 in BMSCs is regulated by miR-144. (A) Expression levels of miR-144, Sfrp1 and Runx2 were detected by reverse transcription-quantitative polymerase chain reaction analysis. (B) Protein expression of Sfrp1, Runx2, CDK4, Wnt1 and $\beta$-catenin was analyzed by western blot analysis. (C) Distributions of Sfrp1 and Runx2 in BMSCs were observed using an immunofluorescence assay (magnification, x200). Data represent the mean \pm SEM. ${ }^{* *} \mathrm{P}<0.01$ and ${ }^{* * *} \mathrm{P}<0.001$. miR, microRNA; BMSCs, bone marrow-derived mesenchymal stem cells; Sfrp1, secreted frizzled-related protein 1; Runx2, Runt-related transcription factor 2; CDK4, cyclin-dependent kinase 4.

The regulation of osteoblast differentiation is a complex process involving multiple genes and multiple pathways, including the Notch signaling pathway, BMP/Smad signaling pathway and Wnt signaling pathway (31-33). The Wnt/ $\beta$-catenin signaling pathway serves a vital role in regulating the proliferation and differentiation of osteoblasts and regeneration of bone tissue. Studies have shown that miRNAs with abnormal expression patterns specifically regulate expression of the Wnt signaling pathway, and are thus closely associated with the occurrence of OP. The expression of miR-218 is regulated by the Wnt pathway. The upregulation of miR-218 inhibits the expression of sclerostin, DKK2 and sFRP2, to create a similar positive feedback regulatory loop leading to osteogenic differentiation (42). Therefore, antagonists of $\mathrm{Wnt} / \beta$-catenin may be useful as novel agents for treating OP. In the present study, it was found that the Wnt/ $\beta$-catenin signaling pathway was significantly activated by the overexpression of miR-144 and sharply suppressed by miR-144 silencing, indicating that miR-144 partially promotes osteoblast differentiation via the Wnt/ $\beta$-catenin signaling pathway. It was further shown that Sfrpl was a target gene of miR-144 and negatively regulated by miR-144 in BMSCs. However, it was found that the miR-144 and Sfrp1 were expressed at high levels in the serum of patients with OP, which may be due to the secretion of miR-144 out of the cell. When miR-144 is secreted out of the cell, such as in the blood, serum detection becomes correspondingly higher. Therefore, a positive regulation was found between miR-144 and Sfrp1 in the serum of patients with OP. Of note, Sfrp1 is an antagonist of the Wnt signaling pathway; it can inhibit the downward transduction of Wnt protein, and thereby inhibit osteoblast activity and osteogenic activity $(43,44)$. In the present study, it was also found that suppression of the $\mathrm{Wnt} / \beta$-catenin signaling pathway as a result of miR-144 knockdown was partially reversed by Sfrp1 siRNA, suggesting that miR-144 may regulate osteoblast differentiation by targeting Sfrp1, which is an antagonist of the Wnt signaling pathway.

During the process by which BMSCs differentiate into osteoblasts, numerous transcription factors become activated or deactivated, and a series of osteoblast-related genes, including Runx2 and Osterix, are expressed $(43,45)$. As an important factor affecting osteoblast differentiation and functional activity, Runx 2 is regulated by BMP, TGF- $\beta$, Wnt and other signaling pathways $(32,46)$. Altering the expression of Runx2 affects the osteogenic differentiation of BMSCs and further influences the formation of normal osteoblasts and bone tissue. In the present study, it was shown that the 
A

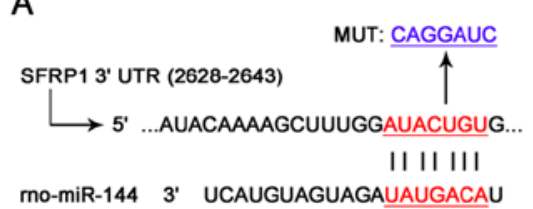

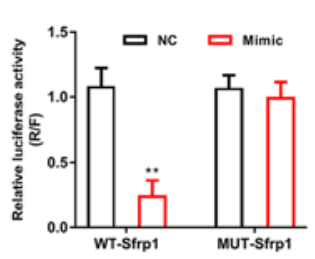

B

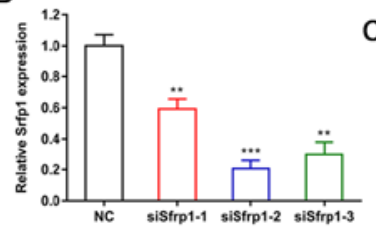

E

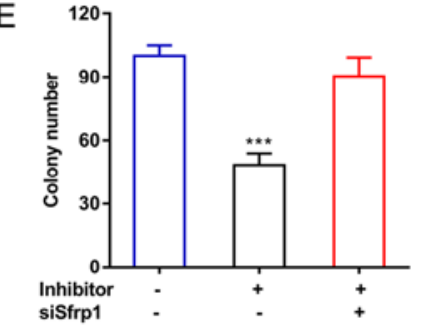

$\mathrm{F}$
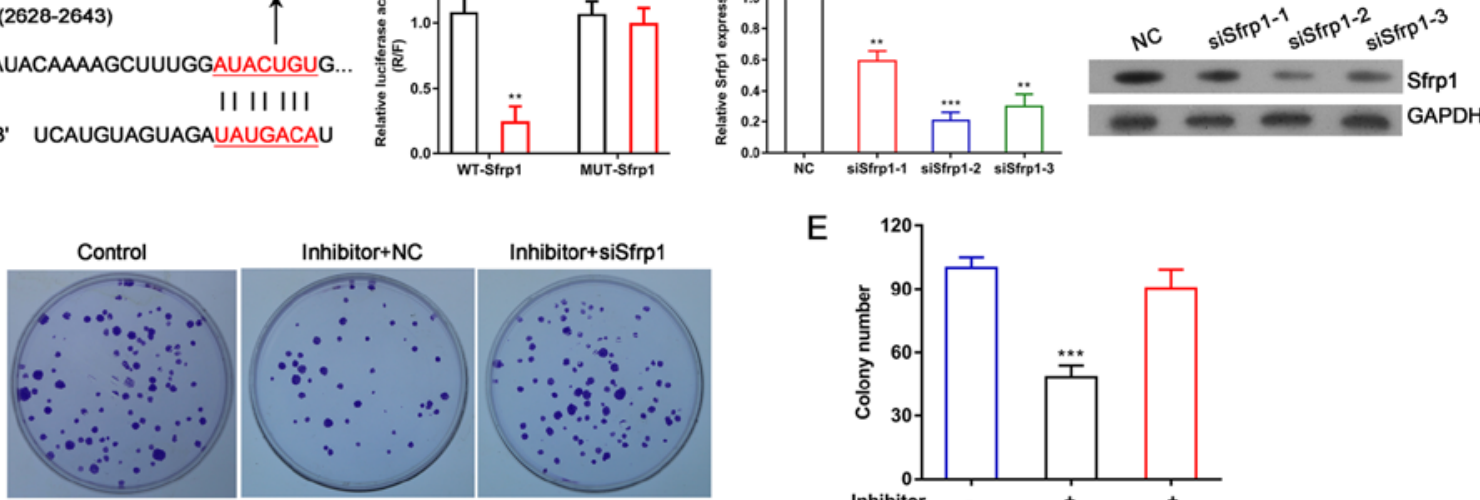

D

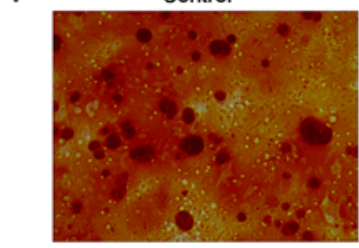

Inhibitor+NC

Inhibitor+siSfrp1

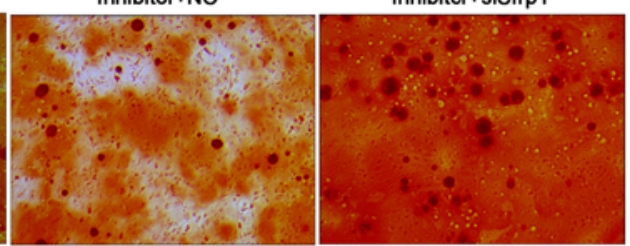

G

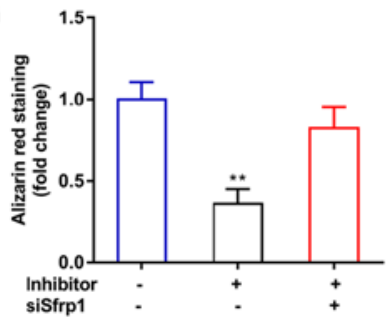

$\mathrm{H}$
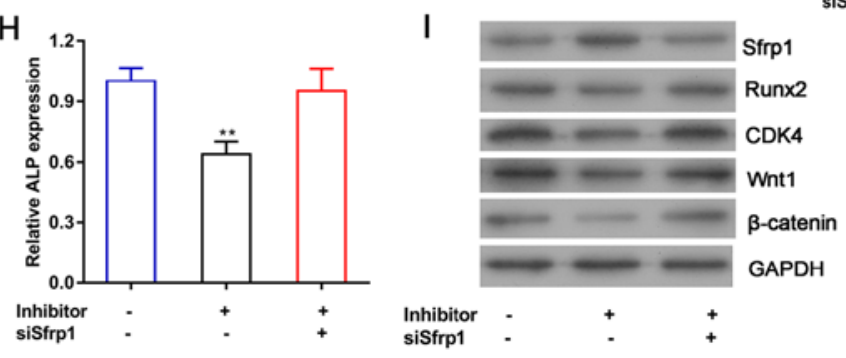

Figure 5. miR-144 promotes the proliferation and differentiation of BMSCs by downregulating the expression of Sfrp1. (A) A luciferase reporter assay was used to examine the direct interaction between miR-144 and Sfrp1. The group of BMSCs were co-transfected with WT Sfrp1 plasmid and miR-144 mimic was compared with the other three groups. BMSCs were transfected with Sfrp1 siRNA, and the interference efficiency was analyzed by (B) reverse transcription-quantitative polymerase chain reaction and (C) western blot analysis, respectively. (D) A colony formation assay was used to determine how Sfrp1 affected the ability of miR-144 to promote BMSC proliferation, and (E) quantified. (F) Alizarin red staining was performed following 3 weeks of osteogenic differentiation to detect the calcium nodi in BMSCs (magnification, x200), with (G) quantification of staining. (H) ALP activity assays were performed following 14 days of osteogenic differentiation. The group of BMSCs co-transfected with the miR-144 inhibitor and NC siRNA was compared with other two groups. (I) Protein expression levels of Sfrp1, Runx2, CDK4, Wnt1 and $\beta$-catenin were analyzed by western blot analysis. GAPDH was used as an internal control. Data represent the mean \pm SEM. ${ }^{* *} \mathrm{P}<0.01$ and ${ }^{* * *} \mathrm{P}<0.001$. miR, microRNA; BMSCs, bone marrow-derived mesenchymal stem cells; WT, wild-type; MUT, mutant; siRNA, small interfering RNA; NC, negative control; ALP, alkaline phosphatase; Sfrp1, secreted frizzled-related protein 1; Runx2, Runt-related transcription factor 2; CDK4, cyclin-dependent kinase 4.

downregulation of miR-144 suppressed the expression of Runx2; however, this effect was partially reversed following treatment with Sfrp1 siRNA, demonstrating that miR-144 promotes the osteoblastic differentiation of BMSCs by targeting Sfrpl. CDK4 is reported to regulate the transition from the $\mathrm{G} 1$ phase to the $\mathrm{S}$ phase, and thus promote the growth of osteoblasts (47). In the present study, it was shown that miR-144 silencing significantly suppressed the expression of CDK4, but that effect was partially reversed when Sfrp1 was simultaneously knocked down. This indicates that miR-144 promotes the proliferation of BMSCs by enhancing the expression of CDK4, and thereby promoting the transition from the G1 phase to the $\mathrm{S}$ phase.

In conclusion, the data obtained in the present study showed that the levels miR-144, Sfrp1 and TNF- $\alpha$ were significantly increased in the serum of patients with OP, and there was a significant positive correlation between miR-144 and Sfrp1. In addition, functional experiments demonstrated that miR-144 promoted the proliferation of BMSCs, inhibited the apoptosis of BMSCs and induced the osteoblastic differentiation of BMSCs, partially by targeting Sfrp1, which is an antagonist of the Wnt signaling pathway. These results provide a basis for investigating the mechanism by which miR-144 is involved in the occurrence and development of OP, and also suggest a novel molecular target for preventing and treating OP.

\section{Acknowledgements}

Not applicable.

\section{Funding}

The present study was supported by the Health and Family Planning Commission of Guangxi Zhuang autonomous region 
(grant no. Z2016387) and the Guilin Scientific Research and Technological Development Project (grant no. 20180106-4-5).

\section{Availability of data and materials}

All data generated or analyzed during the present study are included in this published article.

\section{Authors' contributions}

LT and SL conceived and supervised the study. LT, WL and JH designed and performed experiments. XT and HZ analyzed the data. LT and SL drafted the manuscript and made revisions. All authors read and approved the final manuscript.

\section{Ethics approval and consent to participate}

The collection and analysis of all clinical samples was approved by the Ethics Committee of the Affiliated Hospital of Guilin Medical University (Guilin, China; GLMUIA2016002). The protocols for all animal experiments were approved by the Animal Ethics Committee of the Affiliated Hospital of Guilin Medical University.

\section{Patient consent for publication}

Not applicable.

\section{Competing interests}

The authors declare that they have no competing interests.

\section{References}

1. Janiszewska M, Kulik T, Żołnierczuk-Kieliszek D, Drop B Firlej E and Gajewska I: General self-efficacy level and health behaviours in women over the age of 45 years who have undergone osteoporosis treatment. Prz Menopauzalny 16: 86-95, 2017.

2. Baron R and Gori F: Targeting WNT signaling in the treatment of osteoporosis. Curr Opin Pharmacol 40: 134-141, 2018.

3. Rizou S, Chronopoulos E, Ballas M and Lyritis GP: Clinical manifestations of osteoarthritis in osteoporotic and osteopenic postmenopausal women. J Musculoskelet Neuronal Interact 18 : 208-214, 2018.

4. Nguyen BN, Hoshino H, Togawa D and Matsuyama Y: Cortical thickness index of the proximal femur: A radiographic parameter for preliminary assessment of bone mineral density and osteoporosis status in the age 50 years and over population. Clin Orthop Surg 10: 149-156, 2018.

5. Han Y, You X, Xing W, Zhang Z and Zou W: Paracrine and endocrine actions of bone-the functions of secretory proteins from osteoblasts. osteocytes, and osteoclasts. Bone Res 6: 16, 2018.

6. Gómez-Cerezo N, Casarrubios L, Morales I, Feito MJ, Vallet-Regí M, Arcos D and Portolés MT: Effects of a mesoporous bioactive glass on osteoblasts, osteoclasts and macrophages. J Colloid Interface Sci 528: 309-320, 2018.

7. Mödinger Y, Löffler B, Huber-Lang $M$ and Ignatius A: Complement involvement in bone homeostasis and bone disorders. Semin Immunol 37: 53-65, 2018.

8. Zhang RF, Wang Q, Zhang AA, Xu JG, Zhai LD, Yang XM and Liu XT: Low-level laser irradiation promotes the differentiation of bone marrow stromal cells into osteoblasts through the APN/Wnt/ $\beta$-catenin pathway. Eur Rev Med Pharmacol Sci 22: 2860-2868, 2018.

9. Wang R, Xu B and Xu HG: Up-Regulation of TGF- $\beta$ promotes Tendon-to-Bone healing after anterior cruciate ligament reconstruction using Bone Marrow-Derived mesenchymal stem cells through the TGF- $\beta$ /MAPK signaling pathway in a New Zealand White Rabbit model. Cell Physiol Biochem 41: 213-226, 2017.
10. Xu R, Shi G, Xu L, Gu Q, Fu Y, Zhang P, Cheng J and Jiang H: Simvastatin improves oral implant osseointegration via enhanced autophagy and osteogenesis of BMSCs and inhibited osteoclast activity. J Tissue Eng Regen Med 12: 1209-1219, 2018.

11. Fan JZ, Yang L, Meng GL, Lin YS, Wei BY, Fan J, Hu HM, Liu YW, Chen S, Zhang JK, et al: Estrogen improves the proliferation and differentiation of hBMSCs derived from postmenopausal osteoporosis through notch signaling pathway. Mol Cell Biochem 392: 85-93, 2014.

12. Qiu J, Huang G, Na N and Chen L: MicroRNA-214-5p/TGF- $\beta /$ Smad2 signaling alters adipogenic differentiation of bone marrow stem cells in postmenopausal osteoporosis. Mol Med Rep 17: 6301-6310, 2018.

13. Abdi J, Mutis T, Garssen J and Redegeld FA: Toll-like receptor (TLR)-1/2 triggering of multiple myeloma cells modulates their adhesion to bone marrow stromal cells and enhances bortezomib-induced apoptosis. PLoS One 9: e96608, 2014.

14. Sárközy M, Kahán Z and Csont T: A myriad of roles of miR-25 in health and disease. Oncotarget 9: 21580-21612, 2018.

15. Casciaro M, Di Salvo E, Brizzi T, Rodolico C and Gangemi S: Involvement of miR-126 in autoimmune disorders. Clin Mol Allergy 16: 11, 2018.

16. Pan Y, Jing J, Qiao L, Liu J, An L, Li B, Ren D and Liu W: MiRNA-seq reveals that miR-124-3p inhibits adipogenic differentiation of the stromal vascular fraction in sheep via targeting C/EBPa. Domest Anim Endocrinol 65: 17-23, 2018.

17. Huang C, Yu M and Yao X: MicroRNA-17 and the prognosis of human carcinomas: A systematic review and meta-analysis. BMJ Open 8: e018070, 2018.

18. Lopez-Santillan M, Larrabeiti-Etxebarria A, Arzuaga-Mendez J, Lopez-Lopez E and Garcia-Orad A: Circulating miRNAs as biomarkers in diffuse large B-cell lymphoma: A systematic review. Oncotarget 9: 22850-22861, 2018.

19. Hu X, Tang J, Hu X, Bao P, Pan J, Chen Z and Xian J: MiR-27b impairs adipocyte differentiation of human adipose tissue-derived mesenchymal stem cells by targeting LPL. Cell Physiol Biochem 47: 545-555, 2018.

20. Dai F, Du P, Chang Y, Ji E, Xu Y, Wei C and Li J: Downregulation of MiR-199b-5p inducing differentiation of bone-marrow mesenchymal stem cells (BMSCs) toward Cardiomyocyte-Like cells via HSF1/HSP70 pathway. Med Sci Monit 24: 2700-2710, 2018.

21. Teng Z, Xie X, Zhu Y, Liu J, Hu X, Na Q, Zhang X, Wei G, Xu S, Liu Y, et al: miR-142-5p in bone marrow-derived mesenchymal stem cells promotes osteoporosis involving targeting adhesion molecule VCAM-1 and Inhibiting cell migration. Biomed Res Int 2018: 3274641, 2018.

22. Feichtinger $X$, Muschitz C, Heimel P, Baierl A, Fahrleitner-Pammer A, Redl H, Resch H, Geiger E, Skalicky S, Dormann $\mathrm{R}$, et al: Bone-related circulating MicroRNAs miR-29b-3p, miR-550a-3p, and miR-324-3p and their Association to bone microstructure and histomorphometry. Sci Rep 8: 4867, 2018.

23. Liu Y, Xu F, Pei HX, Zhu X, Lin X, Song CY, Liang QH, Liao EY and Yuan LQ: Vaspin regulates the osteogenic differentiation of MC3T3-E1 through the PI3K-Akt/miR-34c loop. Sci Rep 6: $25578,2016$.

24. Tang X, Lin J, Wang G and Lu J: MicroRNA-433-3p promotes osteoblast differentiation through targeting DKK1 expression. PLoS One 12: e0179860, 2017.

25. Han S, Zhu J and Zhang Y: MiR-144 potentially suppresses proliferation and migration of ovarian cancer cells by targeting RUNX1. Med Sci Monit Basic Res 24: 40-46, 2018.

26. Tao P, Wen H, Yang B, Zhang A, Wu X and Li Q: MiR-144 inhibits growth and metastasis of cervical cancer cells by targeting VEGFA and VEGFC. Exp Ther Med 15: 562-568, 2018.

27. Liu J, Feng L, Zhang H, Zhang J, Zhang Y, Li S, Qin L, Yang Z and Xiong J: Effects of miR-144 on the sensitivity of human anaplastic thyroid carcinoma cells to cisplatin by autophagy regulation. Cancer Biol Ther 19: 484-4962, 2018.

28. Ren YF, Zhang TH, Zhong S, Zhao YT and Lv YN: MiR-144 suppresses proliferation and induces apoptosis of osteosarcoma cells via direct regulation of mTOR expression. Oncol Lett 15: 1163-1169, 2018

29. Hou Q, Huang Y, Zhu S, Li P, Chen X, Hou Z and Liu F: MiR-144 Increases Intestinal Permeability in IBS-D Rats by Targeting OCLN and ZO1. Cell Physiol Biochem 44: 2256-2268, 2017.

30. Xiang C, Cui SP and Ke Y: MiR-144 inhibits cell proliferation of renal cell carcinoma by targeting MTOR. J Huazhong Univ Sci Technolog Med Sci 36: 186-192, 2016 
31. Wu M, Chen G and Li YP: TGF- $\beta$ and BMP signaling in osteoblast, skeletal development, and bone formation, homeostasis and disease. Bone Res 4: 16009, 2016.

32. Nakajima K, Kho DH, Yanagawa T, Harazono Y, Gao X, Hogan V and Raz A: Galectin-3 inhibits osteoblast differentiation through notch signaling. Neoplasia 16: 939-949, 2014

33. Duan P and Bonewald LF: The role of the wnt/ $\beta$-catenin signaling pathway in formation and maintenance of bone and teeth. Int J Biochem Cell Biol 77: 23-29, 2016.

34. Cao F, Zhan J, Chen X, Zhang K, Lai R and Feng Z: MiR-214 promotes periodontal ligament stem cell osteoblastic differentiation by modulating Wnt $/ \beta$-catenin signaling. Mol Med Rep 16: 9301-9308, 2017.

35. Dejaeger M, Böhm AM, Dirckx N, Devriese J, Nefyodova E, Cardoen R, St-Arnaud R, Tournoy J, Luyten FP and Maes C: Integrin-Linked kinase regulates bone formation by controlling cytoskeletal organization and modulating BMP and Wnt signaling in osteoprogenitors. J Bone Miner Res 32: 2087-2102, 2017.

36. Warrier S, Marimuthu R, Sekhar S, Bhuvanalakshmi G, Arfuso F, Das AK, Bhonde R, Martins R and Dharmarajan A: sFRP-mediated Wnt sequestration as a potential therapeutic target for Alzheimer's disease. Int J Biochem Cell Biol 75: 104-111, 2016.

37. Amjadi-Moheb F, Hosseini SR, Kosari-Monfared M, Ghadami E, Nooreddini $\mathrm{H}$ and Akhavan-Niaki $\mathrm{H}$ : A specific haplotype in potential miRNAs binding sites of secreted frizzled-related protein 1 (SFRP1) is associated with BMD variation in osteoporosis. Gene 677: 132-141, 2018.

38. Livak KJ and Schmittgen TD: Analysis of relative gene expression data using real-time quantitative PCR and the 2(-Delta Delta C(T)) method. Methods 25: 402-408, 2001.

39. Chim CS, Pang R, Fung TK, Choi CL and Liang R: Epigenetic dysregulation of Wnt signaling pathway in multiple myeloma. Leukemia 21: 2527-2536, 2007.

40. Shen G, Ren H, Qiu T, Zhang Z, Zhao W, Yu X, Huang J, Tang J, Liang, Yao Z, et al: Mammalian target of rapamycin as a therapeutic target in osteoporosis. J Cell Physiol 233: 3929-3944, 2018.
41. Li X, Ji J, Wei W and Liu L: MiR-25 promotes proliferation, differentiation and migration of osteoblasts by up-regulating Rac1 expression. Biomed Pharmacother 99: 622-628, 2018

42. Hassan MQ, Maeda Y, Taipaleenmaki H, Zhang W, Jafferji M, Gordon JA, Li Z, Croce CM, van Wijnen AJ, Stein JL, et al: MiR-218 directs a Wnt signaling circuit to promote differentiation of osteoblasts and osteomimicry of metastatic cancer cells. J Biol Chem 287: 42084-42092, 2012

43. Holdsworth G, Greenslade K, Jose J, Stencel Z, Kirby H, Moore A, Ke HZ and Robinson MK: Dampening of the bone formation response following repeat dosing with sclerostin antibody in mice is associated with up-regulation of Wnt antagonists. Bone 107: 93-103, 2018.

44. Wang FS, Lin CL, Chen YJ, Wang CJ, Yang KD, Huang YT, Sun YC and Huang HC: Secreted frizzled-related protein 1 modulates glucocorticoid attenuation of osteogenic activities and bone mass. Endocrinology 146: 2415-2423, 2005.

45. Wang C, Liao $\mathrm{H}$ and Cao Z: Role of Osterix and MicroRNAs in bone formation and tooth development. Med Sci Monit 22: 2934-2942, 2016.

46. Stewart S, Gomez AW, Armstrong BE, Henner A and Stankunas K: Sequential and opposing activities of Wnt and BMP coordinate zebrafish bone regeneration. Cell Rep 6: 482-498, 2014

47. Fujita M, Urano T, Horie K, Ikeda K, Tsukui T, Fukuoka H, Tsutsumi O, Ouchi $\mathrm{Y}$ and Inoue S: Estrogen activates cyclin-dependent kinases 4 and 6 through induction of cyclin D in rat primary osteoblasts. Biochem Biophys Res Commun 299: 222-228, 2002.

This work is licensed under a Creative Commons Attribution-NonCommercial-NoDerivatives 4.0 International (CC BY-NC-ND 4.0) License. 